\title{
SOME REMARKS ON THE PAPER "GLOBAL OPTIMIZATION IN METRIC SPACES WITH PARTIAL ORDERS
}

\author{
MOOSA GABELEH* AND JACK MARKIN
}

\begin{abstract}
The aim of this note is to show that the main conclusion of a recent paper by Sadiq Basha [S. Sadiq Basha, Global optimization in metric spaces with partial orders, Optimization, 63 (2014), 817-825] can be obtained as a consequence of corresponding existing results in fixed point theory in the setting of partially ordered metric spaces. Moreover, by a similar approach, we prove that in the paper [V. Pragadeeswarar, M. Marudai, Best proximity points: approximation and optimization in partially ordered metric spaces, Optim. Lett. 7 (2013), 1883-1892] the results are not real generalizations but particular cases of existing fixed point theorems in the literature.
\end{abstract}

MSC(2010): 90C26, 90C30.

Keywords: Partially ordered set; Proximally increasing mapping; Ordered proximal contraction; Best proximity point.

\section{Introduction}

Let $(X, \preceq)$ be a partially ordered set. A self mapping $T: X \rightarrow X$ is said to be monotone nondecreasing if $T(x) \preceq T(y)$ whenever $x, y \in X, x \preceq y$. In 2005 the following fixed point theorem was established by Nieto and Rodri'guez-Lo'pez for monotone nondecreasing mappings which can be considered as an extension of the Banach contraction principle. We will provide a brief proof here since the main ideas will be used in the sequel.

Theorem 1.1. ([1]) Let $(X, \preceq)$ be a partially ordered set and $T: X \rightarrow X$ be a self mapping which is monotone nondecreasing. Assume that there is a metric $d$ on $X$ such that $(X, d)$ is a complete metric space and $X$ satisfies the condition

if a nondecreasing sequence $\left\{x_{n}\right\} \rightarrow x \in X$, then $x_{n} \preceq x, \forall n$.

Suppose that there exists $\alpha \in[0,1[$ such that $d(T x, T y) \leq \alpha d(x, y)$ for every $x, y \in X$ with $x \preceq y$. If there exists $x_{0} \in X$ with $x_{0} \preceq T\left(x_{0}\right)$, then $T$ has

Date: Received: February 11, 2021, Accepted: May 29, 2021.

${ }^{*}$ Corresponding author. 
a fixed point. Moreover, if we define $x_{n}=T x_{n-1}$ for all $n \in \mathbb{N}$, then the sequence $\left\{x_{n}\right\}$ converges to a fixed point of $T$.

Proof. Since $x_{0} \in X$ with $x_{0} \preceq T\left(x_{0}\right)$ and $T$ is monotone nondecreasing, the Picard's iteration sequence $\left\{T^{n}\left(x_{0}\right)\right\}$ is increasing. It now follows from the assumption on the mapping $T$ that there exists $\alpha \in[0,1[$ such that

$$
d\left(T^{n+1} x_{0}, T^{n} x_{0}\right) \leq \alpha d\left(T^{n} x_{0}, T^{n-1} x_{0}\right), \quad \forall n \in \mathbb{N},
$$

that is, $\left\{T^{n}\left(x_{0}\right)\right\}$ is a Cauchy sequence and so converges to an element $p \in X$. By using (1) we conclude that $x_{n} \preceq p$ for all $n \in \mathbb{N}$. We now have

$$
d\left(T^{n+1} x_{0}, T p\right) \leq \alpha d\left(T^{n} x_{0}, p\right) \rightarrow^{(n \rightarrow \infty)} 0,
$$

which ensures that $p$ is a fixed point of $T$.

Throughout this article we denote by $\Psi$ the class of the altering distance functions $\psi:[0, \infty) \rightarrow[0, \infty)$ which satisfy the following conditions:

(i) $\psi$ is continuous and nondecreasing;

(ii) $\psi(t)=0$ if and only if $t=0$.

This class of functions was first introduced in [6].

In [5] Harjani and Sadarangani established the following extension of Theorem 1.1 by using altering distance functions as control functions on contractive conditions.

Theorem 1.2. ([5]) Let $(X, \preceq)$ be a partially ordered set and suppose that there exists a metric $d$ in $X$ such that $(X, d)$ is a complete metric space and $X$ satisfies the condition (1) of Theorem 1.1. Let $T: X \rightarrow X$ be a monotone nondecreasing self mapping such that

$$
\psi(d(T x, T y)) \leq \psi(d(x, y))-\varphi(d(x, y)), \quad \forall x, y \in X \text { with } x \preceq y,
$$

where $\psi, \varphi \in \Psi$. If there exists $x_{0} \in X$ with $x_{0} \preceq T\left(x_{0}\right)$, then $T$ has a fixed point. Moreover, if we define $x_{n}=T x_{n-1}$ for all $n \in \mathbb{N}$, then the sequence $\left\{x_{n}\right\}$ converges to the fixed point of $T$.

Recently, Theorem 1.1 and Theorem 1.2 was generalized in [9] and [7] in order to resolve an optimization problem in the setting of a metric space that is endowed with a partial order.

In this article we show that the results of $[7,9]$ not only are not real extensions of Theorem 1.1, Theorem 1.2 but also they are consequences of Theorem 1.1 and Theorem 1.2, respectively. We refer to [3, 4] for more related subject.

\section{Preliminaries}

Let $(X, d)$ be a metric space equipped with a partial order relation " $\preceq "$ and $(A, B)$ be a pair of nonempty subsets of $X$. We use the following notions and notations in the sequel:

$$
\begin{gathered}
\operatorname{dist}(A, B):=\inf \{d(x, y):(x, y) \in A \times B\}, \\
A_{0}:=\{x \in A: d(x, y)=\operatorname{dist}(A, B), \text { for some } y \in B\},
\end{gathered}
$$


SOME REMARKS ON THE PAPER "GLOBAL OPTIMIZATION IN METRIC SPACES WITH PARTIAL ORDERS

$$
B_{0}:=\{y \in B: d(x, y)=\operatorname{dist}(A, B), \text { for some } x \in A\},
$$

We mention that a point $x^{\star} \in A$ is said to be a best proximity point for a non-self mapping $T: A \rightarrow B$ provided that

$$
d\left(x^{\star}, T x^{\star}\right)=\operatorname{dist}(A, B) .
$$

It is remarkable to note that if $x^{\star} \in A$ is a best proximity point for the nonself mapping $T$, then it is a solution of the following minimization problem: Find

$$
\min _{x \in A} d(x, T x)
$$

Definition 2.1. ([10]) The pair $(A, B)$ is said to have P-property if and only if

$$
\left\{\begin{array}{l}
d\left(x_{1}, y_{1}\right)=\operatorname{dist}(A, B), \\
d\left(x_{2}, y_{2}\right)=\operatorname{dist}(A, B),
\end{array} \Longrightarrow d\left(x_{1}, x_{2}\right)=d\left(y_{1}, y_{2}\right),\right.
$$

where $x_{1}, x_{2} \in A_{0}$ and $y_{1}, y_{2} \in B_{0}$.

Definition 2.2. ([8]) A non-self mapping $T: A \rightarrow B$ is said to be proximally increasing if it satisfies the condition that

$$
\left\{\begin{array}{l}
x_{1} \preceq x_{2}, \\
d\left(u_{1}, T x_{1}\right)=\operatorname{dist}(A, B), \quad \Longrightarrow u_{1} \preceq u_{2}, \\
d\left(u_{2}, T x_{2}\right)=\operatorname{dist}(A, B),
\end{array}\right.
$$

for all $x_{1}, x_{2}, u_{1}, u_{2} \in A$.

Definition 2.3. ([8]) A non-self mapping $T: A \rightarrow B$ is said to be an ordered proximal contraction if there exists a non-negative real number $\alpha<1$ such that

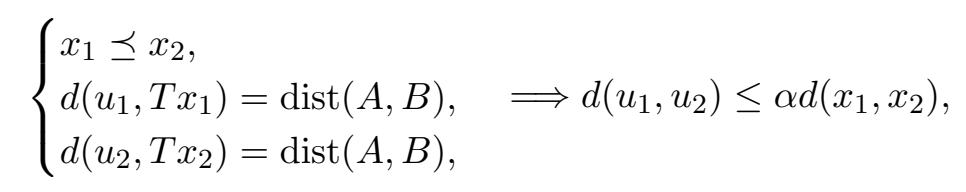

for all $x_{1}, x_{2}, u_{1}, u_{2} \in A$.

Definition 2.4. ([9]) Given non-self mappings $S, T: A \rightarrow B$ the pair $(S ; T)$ is said to be proximally increasing if

$$
\left\{\begin{array}{l}
x \preceq y, \\
d(u, S x)=\operatorname{dist}(A, B), \quad \Longrightarrow u \preceq v, \\
d(v, T y)=\operatorname{dist}(A, B),
\end{array}\right.
$$

for all $x, u \in A, y, v \in B$.

Definition 2.5. ([9]) Given non-self mappings $S, T: A \rightarrow B$ the pair $(S ; T)$ is form an ordered proximal cyclic contraction if there exists a non-negative 
real number $\beta<1$ such that

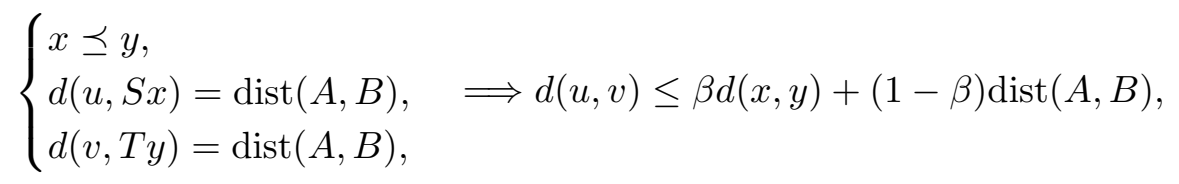

for all $x, u \in A, y, v \in B$.

Here we state the main results of $[7,9]$.

Theorem 2.6. (see Theorem 3.1 of [9]) Let $X$ be a nonempty set such that $(X, \preceq)$ is a partially ordered set and $(X, d)$ is a complete metric space. Let $A$ and $B$ be non-void closed subsets of the metric space $(X, d)$ such that $A_{0}$ is nonempty. Let $S, T: A \rightarrow B$ and $g: A \cup B \rightarrow A \cup B$ satisfy the following conditions:

(i) $S$ and $T$ are proximally increasing, ordered proximal contractions;

(ii) $S\left(A_{0}\right) \subseteq B_{0}$ and $T\left(B_{0}\right) \subseteq A_{0}$;

(iii) $g$ is a surjective isometry, its inverse is an increasing mapping, $A_{0} \subseteq$ $g\left(A_{0}\right)$ and $B_{0} \subseteq g\left(B_{0}\right)$;

(iv) The pair $(S ; T)$ forms a proximally increasing, ordered proximal cyclic contraction.

(v) There exist elements $x_{0}, x_{1} \in A_{0}$ and $y_{0}, y_{1} \in B_{0}$ such that

$$
d\left(g x_{1}, S x_{0}\right)=\operatorname{dist}(A, B)=d\left(g y_{1}, T y_{0}\right),
$$

where $x_{0} \preceq x_{1}, y_{0} \preceq y_{1}$ and $x_{0} \preceq y_{0}$;

(vi) The sets $A$ and $B$ satisfy the condition (1) of Theorem 1.1.

Then there exists an element $\left(x^{\star}, y^{\star}\right) \in A \times B$ such that

$$
d\left(g x^{\star}, S x^{\star}\right)=d\left(g y^{\star}, T y^{\star}\right)=d\left(x^{\star}, y^{\star}\right)=\operatorname{dist}(A, B) .
$$

Further the sequence $\left(\left\{x_{n}\right\},\left\{y_{n}\right\}\right)$ in $A_{0} \times B_{0}$ defined by

$$
d\left(g x_{n+1}, S x_{n}\right)=\operatorname{dist}(A, B)=d\left(g y_{n+1}, T y_{n}\right), \quad \forall n \in \mathbb{N} \cup\{0\},
$$

converges to the element $\left(x^{\star}, y^{\star}\right)$.

Theorem 2.7. (see Theorems 2.1 and 2.2 of [7]) Let $X$ be a nonempty set such that $(X, \preceq)$ is a partially ordered set and $(X, d)$ is a complete metric space. Let $A$ and $B$ be non-void closed subsets of the metric space $(X, d)$ such that $A_{0}$ is nonempty. Let $T: A \rightarrow B$ satisfy the following conditions:

(i) $T$ is a proximally increasing such that $T\left(A_{0}\right) \subseteq B_{0}$ and $(A, B)$ satisfies the P-property;

(ii) there exist elements $x_{0}$ and $x_{1}$ in $A_{0}$ such that

$$
x_{0} \preceq x_{1}, \quad d\left(x_{1}, T x_{0}\right)=\operatorname{dist}(A, B),
$$

(iii) for all $x, y \in A$ with $x \preceq y$,

$$
\psi(d(T x, T y)) \leq \psi(d(x, y))-\varphi(d(x, y)),
$$


where $\varphi, \psi \in \Psi$

(iv) The set A satisfies the condition (1) of Theorem 1.1.

Then $T$ has a best proximity point. Further the sequence $\left\{x_{n}\right\}$ defined by

$$
d\left(x_{n+1}, T x_{n}\right)=\operatorname{dist}(A, B), \quad \forall n \in \mathbb{N} \cup\{0\},
$$

converges to the best proximity point of $T$.

\section{Main results}

Theorem 3.1. Theorem 2.6 is a straightforward consequence of Theorem 1.1 .

Proof. Let $x \in A_{0}$. Since $S x \in B_{0}$, there exists an element $u \in A_{0}$ such that $d(u, S x)=\operatorname{dist}(A, B)$. By the fact that $A_{0} \subseteq g\left(A_{0}\right)$, we can find an element $\hat{u} \in A_{0}$ for which $u=g \hat{u}$ and so $d(g \hat{u}, S x)=\operatorname{dist}(A, B)$. It is worth noticing that if there exists another element $\check{u} \in A_{0}$ for which $d(g \check{u}, S x)=\operatorname{dist}(A, B)$, then by this reality that $S$ is an ordered proximal contraction and $g$ is an isometry, we obtain

$$
d(\hat{u}, \check{u})=d(g \hat{u}, g \check{u}) \leq \alpha d(x, x)=0,
$$

which implies that $\hat{u}=\check{u}$. Thus we can define a self mapping $\Pi_{1}: A_{0} \rightarrow A_{0}$ such that $d\left(g \Pi_{1} x, S x\right)=\operatorname{dist}(A, B)$ for all $x \in A_{0}$. By a similar argument we consider the self mapping $\Pi_{2}: B_{0} \rightarrow B_{0}$ for which $d\left(g \Pi_{2} y, T y\right)=\operatorname{dist}(A, B)$ for any $y \in B_{0}$. We have the following observations about the mappings $\Pi_{i}$ for $i \in\{1,2\}$.

\& Let $x_{1}, x_{2} \in A_{0}$ be such that $x_{1} \preceq x_{2}$. Then

$$
\left\{\begin{array}{l}
d\left(g \Pi_{1} x_{1}, S x_{1}\right)=\operatorname{dist}(A, B), \\
d\left(g \Pi_{1} x_{2}, S x_{2}\right)=\operatorname{dist}(A, B) .
\end{array}\right.
$$

Since $S$ is a proximally increasing, $g \Pi_{1} x_{1} \preceq g \Pi_{1} x_{2}$. Since $g^{-1}$ is increasing, we must have $\Pi_{1} x_{1} \preceq \Pi_{1} x_{2}$, that is, $\Pi_{1}$ is monotone nondecreasing. Equivalently, we can see that $\Pi_{2}$ is also monotone nondecreasing.

\& Let $x_{1}, x_{2} \in A_{0}$ be such that $x_{1} \preceq x_{2}$. Then

$$
\left\{\begin{array}{l}
d\left(g \Pi_{1} x_{1}, S x_{1}\right)=\operatorname{dist}(A, B), \\
d\left(g \Pi_{1} x_{2}, S x_{2}\right)=\operatorname{dist}(A, B) .
\end{array}\right.
$$

Since $S$ is an ordered proximal contraction, there exists $\alpha \in[0,1)$ such that

$$
d\left(\Pi_{1} x_{1}, \Pi_{1} x_{2}\right)=d\left(g \Pi_{1} x_{1}, g \Pi_{1} x_{2}\right) \leq \alpha d\left(x_{1}, x_{2}\right) .
$$

Similarly, if $y_{1}, y_{2} \in B_{0}$ with $y_{1} \preceq y_{2}$, then

$$
d\left(\Pi_{2} y_{1}, \Pi_{2} y_{2}\right) \leq \alpha d\left(y_{1}, y_{2}\right) .
$$

as By the assumption $(v)$ of Theorem 2.6, there exist $x_{0}, x_{1} \in A_{0}$ and $y_{0}, y_{1} \in B_{0}$ with $x_{0} \preceq x_{1}$ and $y_{0} \preceq y_{1}$ such that $d\left(g x_{1}, S x_{0}\right)=\operatorname{dist}(A, B)=$ $d\left(g y_{1}, T y_{0}\right)$. Besides, by the definition of the mapping $\Pi_{1}$, we have $d\left(g \Pi_{1} x_{0}, S x_{0}\right)=$ $\operatorname{dist}(A, B)$. Because of the fact that $S$ is an ordered proximal contraction, we 
conclude that $x_{1}=\Pi_{1} x_{0}$ and so $x_{0} \preceq \Pi_{1} x_{0}$. Similarly, we obtain $y_{0} \preceq \Pi_{2} y_{0}$.

\& Now define the mapping $\Pi: A_{0} \cup B_{0} \rightarrow A_{0} \cup B_{0}$ with

$$
\Pi z= \begin{cases}\Pi_{1} z & \text { if } z \in A_{0}, \\ \Pi_{2} z & \text { if } z \in B_{0} .\end{cases}
$$

Then $\Pi\left(A_{0}\right) \subseteq A_{0}$ and $\Pi\left(B_{0}\right) \subseteq B_{0}$, that is, $\Pi$ is noncyclic on $A_{0} \cup B_{0}$. Let $(x, y) \in A_{0} \times B_{0}$ be such that $x \preceq y$. Then we have

$$
\left\{\begin{array}{l}
d(g \Pi x, S x)=\operatorname{dist}(A, B), \\
d(g \Pi y, T y)=\operatorname{dist}(A, B) .
\end{array}\right.
$$

Since the pair $(S ; T)$ forms an ordered proximal cyclic contraction, we obtain

$$
d(\Pi x, \Pi y)=d\left(g \Pi_{1} x, g \Pi_{2} y\right) \leq \beta d(x, y)+(1-\beta) \operatorname{dist}(A, B) .
$$

as For the considered elements $\left(x_{0}, y_{0}\right),\left(x_{1}, y_{1}\right) \in A_{0} \times B_{0}$ which satisfy the condition $(v)$ since $x_{0} \preceq \Pi_{1} x_{0}$ and $\Pi_{1}$ is monotone nondecreasing, the sequence $\left\{\Pi_{1}^{n} x_{0}\right\}$ is increasing. Similarly, the sequence $\left\{\Pi_{2}^{n} y_{0}\right\}$ is also increasing. It now follows from the proof of Theorem 1.1 that the sequences $\left\{\Pi_{1}^{n} x_{0}\right\}$ and $\left\{\Pi_{2}^{n} y_{0}\right\}$ are Cauchy. Let $\left(x^{\star}, y^{\star}\right) \in A \times B$ be such that

$$
\Pi_{1}^{n} x_{0} \rightarrow x^{\star}, \quad \Pi_{2}^{n} y_{0} \rightarrow y^{\star} .
$$

If we prove that $\left(x^{\star}, y^{\star}\right) \in A_{0} \times B_{0}$ then by a similar argument of the proof of Theorem 1.1 we deduce that $x^{\star}$ and $y^{\star}$ are the fixed points of $\Pi_{1}$ and $\Pi_{2}$, respectively. To show this, we note that since $x_{0} \preceq y_{0}$ we have

$$
d\left(\Pi x_{0}, \Pi y_{0}\right) \leq \beta d\left(x_{0}, y_{0}\right)+(1-\beta) \operatorname{dist}(A, B) .
$$

Since

$$
\left\{\begin{array}{l}
d\left(g \prod x_{0}, S x_{0}\right)=\operatorname{dist}(A, B), \\
d\left(g \Pi y_{0}, T y_{0}\right)=\operatorname{dist}(A, B),
\end{array}\right.
$$

and the pair $(S ; T)$ forms a proximally increasing, we conclude that $g \Pi x_{0} \preceq$ $g \Pi y_{0}$. By the fact that $g^{-1}$ is increasing, $\Pi x_{0} \preceq \Pi y_{0}$. Again, since the pair $(S ; T)$ forms an ordered proximal cyclic contraction, we obtain

$$
\begin{aligned}
d\left(\Pi^{2} x_{0}, \Pi^{2} y_{0}\right) & \leq \beta d\left(\Pi x_{0}, \Pi y_{0}\right)+(1-\beta) \operatorname{dist}(A, B) \\
& \leq \beta^{2} d\left(x_{0}, y_{0}\right)+\left(1-\beta^{2}\right) \operatorname{dist}(A, B) .
\end{aligned}
$$

Continuing this process and by induction, we conclude that

$$
d\left(\Pi^{n} x_{0}, \Pi^{n} y_{0}\right) \leq \beta^{n} d\left(x_{0}, y_{0}\right)+\left(1-\beta^{n}\right) \operatorname{dist}(A, B) .
$$

Letting $n \rightarrow \infty$ in above inequality, we obtain $d\left(x^{\star}, y^{\star}\right)=\operatorname{dist}(A, B)$, that is, $\left(x^{\star}, y^{\star}\right) \in A_{0} \times B_{0}$. Hence,

$$
\begin{aligned}
d\left(g x^{\star}, S x^{\star}\right) & =d\left(g \Pi_{1} x^{\star}, S x^{\star}\right)=\operatorname{dist}(A, B), \\
d\left(g y^{\star}, T y^{\star}\right) & =d\left(g \Pi_{2} y^{\star}, T y^{\star}\right)=\operatorname{dist}(A, B), \\
d\left(x^{\star}, y^{\star}\right) & =\operatorname{dist}(A, B) .
\end{aligned}
$$


Finally, if for each $n \in \mathbb{N}$ we set $x_{n}=\Pi^{n} x_{0}$ and $y_{n}=\Pi^{n} y_{0}$, then

$$
\begin{gathered}
d\left(g x_{n+1}, S x_{n}\right)=\operatorname{dist}(A, B), \\
d\left(g y_{n+1}, T y_{n}\right)=\operatorname{dist}(A, B), \\
\left(x_{n}, y_{n}\right) \rightarrow\left(x^{\star}, y^{\star}\right) .
\end{gathered}
$$

Theorem 3.2. Theorem 2.7 is a straightforward consequence of Theorem 1.2.

Proof. Since the pair $(A, B)$ has the P-property, it follows from Lemma 3.1 of [2] that both $A_{0}$ and $B_{0}$ are closed. Moreover, if $x \in A_{0}$, then there exists an element $v \in B_{0}$ such that $d(x, v)=\operatorname{dist}(A, B)$. We note that if there is another element $v^{\prime} \in B_{0}$ for which $d\left(x, v^{\prime}\right)=\operatorname{dist}(A, B)$, then from the fact that $(A, B)$ has the P-property, we must have $v=v^{\prime}$. So, we can define a mapping $g: A_{0} \rightarrow B_{0}$ such that

$$
d(x, g x)=\operatorname{dist}(A, B), \quad \forall x \in A_{0} .
$$

It is worth noticing that for any $u_{1}, u_{2} \in A_{0}$, we have $d\left(u_{1}, g u_{1}\right)=\operatorname{dist}(A, B)=$ $d\left(u_{2}, g u_{2}\right)$ which ensures that

$$
d\left(u_{1}, u_{2}\right)=d\left(g u_{1}, g u_{2}\right), \quad \forall u_{1}, u_{2} \in A_{0},
$$

that is, $g$ is an isometry. Hence, $g$ is a bijective isometry mapping. Now consider the self-mapping $g^{-1} T: A_{0} \rightarrow A_{0}$. Here, we check the conditions of Theorem 1.1 for the self mapping $g^{-1} T: A_{0} \rightarrow A_{0}$.

ه Let $x, y \in A_{0}$ be such that $x \preceq y$. Since $g^{-1}$ is an isometry, we conclude that

$$
\psi\left(d\left(\left(g^{-1} T\right) x,\left(g^{-1} T\right) y\right)\right)=\psi(d(T x, T y)) \leq \psi(d(x, y))-\varphi(d(x, y)),
$$

where $\varphi, \psi \in \Psi$.

\$ It follows from the assumption (ii) of Theorem 2.7 that there exist the elements $x_{0}, x_{1} \in A_{0}$ such that $x_{0} \preceq x_{1}$ and $d\left(x_{1}, T x_{0}\right)=\operatorname{dist}(A, B)$. By the fact that $d\left(x_{1}, g x_{1}\right)=\operatorname{dist}(A, B)$ and that $(A, B)$ has the P-property, we obtain $g x_{1}=T x_{0}$ and so, $x_{1}=\left(g^{-1} T\right) x_{0}$ which implies that

$$
x_{0} \preceq\left(g^{-1} T\right) x_{0} .
$$

\$ Let $x, y \in A_{0}$ be such that $x \preceq y$. Since $T\left(A_{0}\right) \subseteq B_{0}$ there are two points $u, v \in A_{0}$ such that

$$
d(u, T x)=\operatorname{dist}(A, B)=d(v, T y) .
$$

Because $T$ is proximally increasing, we must have $u \preceq v$. Besides, from the definition of the mapping $g$ we have $g u=T x$ and $g v=T y$ and hence

$$
\left(g^{-1} T\right) x=u \preceq v=\left(g^{-1} T\right) y,
$$

which implies that the self mapping $g^{-1} T$ is monotone nondecreasing.

Thereby, all of the assumptions of Theorem 1.1 hold and the self mapping 
$g^{-1} T: A_{0} \rightarrow A_{0}$ has a fixed point, called $x^{\star} \in A_{0}$, that is, $g^{-1} T x^{\star}=x^{\star}$ which ensures that $T x^{\star}=g x^{\star}$. Hence,

$$
d\left(x^{\star}, T x^{\star}\right)=d\left(x^{\star}, g x^{\star}\right)=\operatorname{dist}(A, B) .
$$

On the other hand if we define $x_{n}=\left(g^{-1} T\right) x_{n-1}$ for any $n \in \mathbb{N}$, then $x_{n} \rightarrow x^{\star}$. In this case we have $g x_{n}=T x_{n-1}$ and so

$$
d\left(x_{n}, T x_{n-1}\right)=d\left(x_{n}, g x_{n}\right)=\operatorname{dist}(A, B),
$$

and the result follows.

\section{Concluding Remarks}

It was proved by Sadiq Basha that in the setting of compete partially ordered metric spaces a pair of ordered proximal contractions which are proximally increasing has a common best proximity point (see Theorem 2.6). Moreover, an existence and convergence result of a best proximity point for proximally increasing nonself mappings was established by Pragadeeswarar and Maruda using a geometric concept of P-property (see Theorem 2.7).

We have proved that these existence results are straightforward consequences of Theorem 1.1 and Theorem 1.2, respectively.

\section{REFERENCES}

[1] Nieto, J.J., Rodri'guez-Lo'pez, R., Contractive mapping theorems in partially ordered sets and applications to ordinary differential equations, Order, 22 (2005), 223-239.

[2] Gabeleh, M.: Proximal weakly contractive and proximal nonexpansive non-selfmappings in metric and Banach spaces, J. Optim. Theory Appl., 158 (2013), 615-625.

[3] Gabeleh, M., Markin, J.: A note on the paper "Best proximity point results for p-proximal contractions, Acta Math. Hungar., (2021), doi.org/10.1007/s10474-02101139-5.

[4] Gabeleh, M., Vetro, C.: A note on best proximity point theory using proximal contractions, J. Fixed Point Theory Appl., 20 (2018), doi.org/10.1007/s11784-018-0624-4.

[5] Harjani, J., Sadarangani, K.: Generalized contractions in partially ordered metric spaces and applications to ordinary differential equations, Nonlinear Anal., 72 (2010), 1188-1197.

[6] Khan,M.S., Swaleh,M., Sessa, S.: Fixed point theorems by altering distances between the points, Bull. Austral. Math. Soc., 30 (1984), 1-9.

[7] Pragadeeswarar, V., Marudai, M.: Best proximity points: approxi- mation and optimization in partially ordered metric spaces, Optim. Lett., 7 (2013), 1883-1892.

[8] Sadiq Basha, S., Discrete optimization in partially ordered sets, J. Global Optim., 54 (2012), 511-517.

[9] Sadiq Basha, S., Global optimization in metric spaces with partial orders, Optimization, 63 (2014), 817-825.

[10] Sankar Raj, V., A best proximity point theorem for weakly contractive non-selfmappings, Nonlinear Anal., 74 (2011), 4804-4808.

(Moosa Gabeleh) Department of Mathematics, Ayatollah Boroujerdi UniverSITY, BOROUJERD, IRAN.

Email address: Gabeleh@abru.ac.ir

(Jack Markin) 1440 8Th ST. GOLDEN, Co 80401, USA; JMARKIN@NEWMEXICO.COM

Email address: jmarkin@newmexico.com 